\title{
Hay insuficiente evidencia sobre la eficacia de vitamina B-6 en el tratamiento de síndrome premenstrual
}

Efficacy of vitamin B-6 in the treatment of premenstrual syndrome: systematic review. Wyatt KM, Dimmock PW, Jones PW, et al. BMJ 1999; 318: 1375-81.

\section{Objetivo}

Revisar la evidencia científica acerca de la eficacia de la vitamina B-6, en el tratamiento del síndrome premenstrual.

\section{Fuente y selección de datos}

Los autores limitaron la búsqueda a estudios clínicos controlados, randomizados y doble ciego, publicados y no publicados que compararan la efectividad de la vitamina B-6 vs. placebo en el tratamiento del síndrome premenstrual. Se incluyeron pacientes con síntomas premenstruales. Revisaron las siguientes bases de datos: Embase 1988-1996; Medline 1966-1998; Psychlit 1974-1997; Cinahl 19821997 y Cochrane. El análisis de los datos, extraídos de los estudios seleccionados fue realizado independientemente por dos de los autores. Los resultados del metaanálisis se expresaron en OR (Odds Ratios*). Para evaluar la calidad metodológica de los estudios se utilizó una escala de calidad (Jadad Quality Score), con rango entre 0 (mala calidad) y 6 (excelente calidad).

\section{Resultados}

Se identificaron 25 ensayos publicados, de los cuales 9 (2 que evaluaban específicamente la mastalgia premenstrual y 7 con síntomas generales) se incluyeron en el metaanálisis. La metodología global de los ensayos fue pobre, solo tres tuvieron un score de calidad igual a 3 (considerado como aceptable). El tamaño de la muestra varió entre 21 y 434 pacientes, ninguno de los ensayos tuvo en cuenta el poder estadístico* en el cálculo del $n^{*}$. Sólo dos de los estudios mencionan la toma de anticonceptivos orales por parte de Las pacientes. En general, la vitamina B-6 comparada con placebo, mejoró los síntomas premenstruales ( 0 R global 2,32; IC 95\% 1,95$2,54)$; y los síntomas depresivos (OR global 1,69; IC 95\% 1,39$2,06)$ en cuatro ensayos con 541 mujeres.

\section{Conclusiones}

Las conclusiones están limitadas por la baja calidad de la mayoría de los ensayos incluidos. Los resultados sugieren que es probable que dosis de 50 a $100 \mathrm{mg} /$ día de vitamina B-6 sea beneficiosa para tratar los síntomas generales y la depresión premenstruales. Queda en evidencia la necesidad de realizar un ensayo controlado y aleatorizado, con suficiente poder y calidad para poder realizar una recomendación definitiva acerca del uso de la vitamina B-6 en el tratamiento del síndrome premenstrual.

\section{COMENTARIO}

El síndrome premenstrual es transcultural y afecta a mujeres de todas las razas, niveles socioeconómicos y profesiones. De 70 a $90 \%$ de la población femenina padece problemas menstruales recurrentes ${ }^{1}$. Entre 20 y $40 \%$ manifiesta disfunción pasajera mental y física; y 2 a $5 \%$ refiere incapacidad transitoria. Aparece recurrentemente de siete a catorce días antes del período menstrual y remite con la menstruación. Se estima que 10 a $20 \%$ de las mujeres solicita ayuda profesional por síntomas del síndrome premenstrual. Desde que Frank ${ }^{2} e n$ 1931 describió por primera vez la tensión premenstrual, existe mucha controversia a cerca de su abordaje y tratamiento. Para resolver el dilema sobre la eficacia de la vitamina B-6 en los síntomas premenstruales se revisaron estos estudios clínicos (con dispares diseños), con bajo poder estadístico* y de pobre calidad metodológica. Las antedichas características determinan que la combinación de sus resultados para obtener un único resultado sea cuestionable. Este metaanálisis resalta la necesidad de realizar un ensayo controlado, aleatorizado y doble

*Ver Glosario

\section{Dra. Nanci C. Giraudo}

Unidad de Medicina Familiar y Preventiva. Hospital Italiano de Buenos Aires. ciego para determinar si el uso de vitamina B-6 es efectivo en el desorden premenstrual y cual es la dosis recomendada. Algunos estudios muestran que dosis superiores a $200 \mathrm{mg} /$ día de esta vitamina podrían asociarse a neuropatía periférica por toxicidad a la piridoxina ${ }^{3}$. ¿Qué podemos hacer entonces frente a una mujer que nos consulta por síndrome premenstrual? La mayoría de las alternativas terapéuticas actuales son empíricas e incluyen las siguientes: reaseguro de la benignidad de la condición; llevar un diario de los síntomas ya que en el síndrome premenstrual estos ocurren con una temporalidad específica; dieta rica en carbohidratos complejos; programa de ejercicio regular; progesterona administrada durante la fase lutea; y benzodiacepinas de acción corta (a evitar por el alto potencial adictivo) ${ }^{4}$. La mejor evidencia (grado I)* avala a los inhibidores de la recaptación de serotonina como la fluoxetina a $20 \mathrm{mg}$. Estos son efectivos para el alivio de la tensión, la irritabilidad y la disforia con pocos efectos adversos 5 .

\footnotetext{
Referencias

1. Reid RW and Yen SC. Premenstrual Syndrome. Am J Obstet Gynecol 1981; 139: 85

2. Frank RT. Hormonal causes of premenstrual tension. Arc Neurol Psychiatry 193: 26: 1053.

3. London RS, Bradley L, Chiamori NY. Effect of a nutritional supplement on premenstrual syndrome: double blind longitudinal study. J Am Coll Nutr 1991; 10: 40-5.

4. Premenstrual syndrome, en Current Medical Diagnosis and Treatment. 38th edition. Appleton \& Lange 1999.

5. Steiner M et al. Fluoxetine in the treatment of premenstrual dysphoria. N Engl J Med 1995;322:1529.
} 